\title{
Error Rate Analysis for Bit-Loaded Coded OFDM
}

\author{
Mohammad Mohammadnia-Avval, Chris Snow, and Lutz Lampe \\ Department of Electrical \& Computer Engineering \\ University of British Columbia, Vancouver, Canada \\ \{ mohammadm, csnow, lampe \}@ece.ubc.ca
}

\begin{abstract}
Bit-loaded Orthogonal Frequency Division Multiplexing (OFDM) with convolutional coding is a powerful technique for transmission over quasi-static frequency-selective fading channels. Motivated by the lack of appropriate error rate analysis techniques for this popular type of system and channel model, we develop a novel analytical method for bit error rate (BER) estimation of bit-loaded coded OFDM systems operating over frequency-selective quasi-static channels with nonideal interleaving. To illustrate the application of the proposed analysis, we compare the performance of a number of OFDM bit-loading schemes applied to Multiband OFDM (MB-OFDM) for Ultra-Wideband (UWB) radio, and to IEEE 802.11a/g systems for wireless local area networks (WLANs). We also propose and evaluate a hybrid loading scheme which selects the best loading for each channel realization from amongst a number of candidates.
\end{abstract}

\section{INTRODUCTION}

Multicarrier communication systems based on Orthogonal Frequency Division Multiplexing (OFDM) have gained interest from the communications community in recent years, as evidenced by standards such as XDSL (digital subscriber lines), IEEE 802.11a/g for Wireless Local Area Networks (WLANs) [1], IEEE 802.16 (broadband wireless access), and ECMA Multiband OFDM (MB-OFDM) for high-rate UltraWideband (UWB) [2]. In general, the frequency-selective channel for these systems can be assumed to be very slowly time-varying relative to the transmission rate of the device, and can be approximated as quasi-static for the duration of one or more packet transmissions.

Given the frequency-selective quasi-static conditions present in many OFDM systems, it is beneficial to employ bit-loading algorithms to select non-identical modulation schemes for each OFDM subcarrier based on the channel conditions. Bit-loading techniques can be used for either (a) increasing the total throughput by maximizing the sum data rate, or (b) decreasing the error probability and/or transmit power by keeping the total rate fixed and adjusting the modulation per subcarrier in order to take advantage of those subcarriers with the best channel gains. In this work, we focus on the latter class of error rate minimizing loading schemes, of which a number of different algorithms exist [3]-[7].

In addition, most OFDM systems employ channel coding techniques such as bit-interleaved coded modulation (BICM) [8] with convolutional codes in order to mitigate the effects of the fading channel. Simulation-based approaches to obtain system performance in this setting are very time consuming due to the necessity of simulating the system over a large number of channel realizations, thus, there is an interest in analytical methods for evaluating the performance of bitloaded BICM-OFDM operating over quasi-static frequencyselective fading channels. There are well-known techniques for bounding the performance of convolutionally-encoded transmission over many types of fading channels, e.g. [8], [9]. However, such classical bit error rate (BER) analysis techniques are not applicable to the OFDM systems mentioned above for several reasons. Firstly, the short-length channelcoded packet-based transmissions are non-ideally interleaved, which results in non-zero correlation between adjacent coded bits. Secondly, and more importantly, the quasi-static nature limits the number of distinct channel gains to the (relatively small) number of OFDM subcarriers. This small number of distinct channel gains must not be approximated by the full fading distribution for a valid performance analysis, as would be the case in a fast-fading channel. Recent work has developed a pairwise error probability (PEP) analysis for loaded coded OFDM [10]. However, this analysis assumes the use of a random interleaver, and furthermore the resultant expressions are highly complex and are not amenable to numerical evaluation.

In this paper, we develop a novel analysis technique for bit-loaded coded OFDM systems (Section III), extending recent results for non-loaded coded OFDM [11]. This method provides system designers with a simple way to study the performance of different bit-loading and channel coding schemes without resorting to lengthly simulations. For illustration, we compare the performance of a number of OFDM bit-loading schemes applied to MB-OFDM UWB and to IEEE 802.11a/g systems (Section IV). We also propose and evaluate a hybrid loading scheme which selects the best loading for each channel realization from amongst a number of candidates.

Notation: In this paper, $\boldsymbol{x}$ and $\boldsymbol{X}$ denote a vector and a matrix, respectively, $[\cdot]^{T}$ denotes vector transposition, $\operatorname{diag}(\boldsymbol{x})$ denotes a matrix with the elements of $\boldsymbol{x}$ on the main diagonal, $\mathbb{C}$ denotes the field of complex numbers, $\oplus$ denotes elementwise XOR, and $Q(\cdot)$ is the Gaussian Q function [9].

\section{SySTEM MODEL}

Throughout this paper we consider an $N$-subcarrier OFDM system employing a general bit-loading scheme, which selects a $2^{m_{i}}$-ary QAM $\left(2^{m_{i}}\right.$-QAM) modulation for subcarrier $1 \leq i \leq N$ based on the channel conditions. ${ }^{1}$ We denote

\footnotetext{
${ }^{1}$ With a slight abuse of notation, we use the term 2-QAM to denote binary phase shift keying (BPSK).
} 
the average number of bits per modulated symbol by $\bar{m}$. The particular loading algorithms applied will be discussed in Section IV.

The system employs a (possibly punctured) convolutional code of rate $R_{c}$. We assume that the transmitter selects a vector of $R_{c} N \bar{m}$ random message bits for transmission, denoted by

$$
\boldsymbol{b}=\left[\begin{array}{llll}
b_{1} & b_{2} & \ldots & b_{R_{c} N \bar{m}}
\end{array}\right]^{T} .
$$

The messages bits are convolutionally encoded by the mapping

$$
\mathcal{C}:\{0,1\}^{R_{c} N \bar{m}} \rightarrow\{0,1\}^{L_{c}}
$$

to produce the vector

$$
\boldsymbol{c}=\mathcal{C}(\boldsymbol{b})
$$

of length $L_{c}=N \bar{m}$. The vector $c$ is then interleaved by the mapping

$$
\pi:\{0,1\}^{L_{c}} \rightarrow\{0,1\}^{L_{c}}
$$

to produce the vector

$$
\boldsymbol{c}^{\pi}=\pi(\boldsymbol{c})
$$

of length $L_{c}$. The interleaved bits $c^{\pi}$ are finally modulated using $2^{m_{i}}$-QAM on subcarrier $1 \leq i \leq N$, where the modulation is represented by the mapping

$$
\mathcal{M}_{\boldsymbol{h}}:\{0,1\}^{L_{c}} \rightarrow \mathbb{C}^{N}
$$

resulting in the $N$ modulated symbols denoted by the vector

$$
\boldsymbol{x}=\left[\begin{array}{llll}
x_{1} & x_{2} & \ldots & x_{N}
\end{array}\right]^{T}=\mathcal{M}_{\boldsymbol{h}}\left(\boldsymbol{c}^{\pi}\right) .
$$

It is important to note the dependence of $\mathcal{M}_{h}$ on the frequency-domain channel gains $\boldsymbol{h}=\left[\begin{array}{llll}h_{1} & h_{2} & \ldots & h_{N}\end{array}\right]$ as a result of the channel gain dependent loading algorithms. For a particular $h$, the mapping $\mathcal{M}_{\boldsymbol{h}}$ is obtained by running the chosen loading algorithm in order to select the modulation for each subcarrier.

We will assume that the OFDM system is designed such that the cyclic prefix is longer than the channel impulse response. Thus, we can equivalently consider the channel in the frequency domain, and writing $\boldsymbol{H}=\operatorname{diag}(\boldsymbol{h})$, we can express the received symbols as

$$
\boldsymbol{r}=\sqrt{E_{s}} \boldsymbol{H} \boldsymbol{x}+\boldsymbol{n},
$$

where $\boldsymbol{n}$ is a vector of independent complex additive white Gaussian noise (AWGN) variables with variance $\mathcal{N}_{0}$ and $E_{s}$ is the energy per modulated symbol. The energy per information bit is $E_{b}=E_{s} /\left(R_{c} \bar{m}\right)$.

We assume perfect timing and frequency synchronization. The receiver employs a soft-output detector followed by deinterleaving, depuncturing, and Viterbi decoding, resulting in an estimate

$$
\hat{\boldsymbol{b}}=\left[\begin{array}{llll}
\hat{b}_{1} & \hat{b}_{2} & \ldots & \hat{b}_{R_{c} N \bar{m}}
\end{array}\right]^{T}
$$

of the original transmitted information bits.

\section{PERFormAnCE ANALYSis}

In this section, we present a method for approximating the performance of bit-loaded coded multicarrier systems operating over frequency-selective, quasi-static fading channels, based on approximating the performance of the system over individual channel realizations. This method is based on considering the set of error vectors, introduced below.

One major problem in the analysis of $2^{m}$-QAM modulation schemes with $2^{m}>4$ is that the probability of error for a given bit depends on the whole transmitted symbol (i.e., it also depends on the other bits in the symbol). For this reason, for the combination of convolutional coding and $2^{m}$ QAM it is not sufficient to adopt the classical approach of considering deviations from the all-zero codeword. In theory, one must average over all possible choices for $\boldsymbol{c}$. Since this is computationally intractable, we simply assume the transmitted information bits $\boldsymbol{b}$ (and hence $\boldsymbol{x}$ ) are chosen randomly. For 4-QAM (where the joint linearity of code and modulator is maintained) this is exactly equivalent to considering an allzero codeword. In the case of $2^{m}>4$, we have verified for the two analysis methods proposed below that the results are practically invariant to the choice of $\boldsymbol{b}$.

\section{A. Set of Error Vectors}

Consider a convolutional encoder initialized to the all-zero state, where the reference (correct) codeword is the all-zero codeword. We construct all $L$ input sequences which cause an immediate deviation from the all-zero state (i.e., those whose first input bit is 1) and subsequently return the encoder to the all-zero state with an output Hamming weight of at most $w_{\max }$. Let $\mathcal{E}$ be the set of all vectors $\boldsymbol{e}_{\ell}(1 \leq \ell \leq L)$ representing the output sequences (after puncturing) associated with these input sequences, i.e., $\mathcal{E}=\left\{\boldsymbol{e}_{1}, \boldsymbol{e}_{2}, \ldots, \boldsymbol{e}_{L}\right\}$. Let $l_{\ell}$ be the length of $\boldsymbol{e}_{\ell}$ (the number of output bits after puncturing), and let $a_{\ell}$ be the Hamming weight of the input associated with $\boldsymbol{e}_{\ell}$. Note that the choice of $\omega_{\max }$ governs the value of $L$ (i.e., once the maximum allowed Hamming weight is set, the number of error events $L$ is known).

We term $e_{\ell}$ an "error vector" and $\mathcal{E}$ the set of error vectors. The set $\mathcal{E}$ contains all the low-weight error events, which are the most likely deviations in the trellis. As with standard union-bound techniques for convolutional codes [9], the lowweight terms will dominate the error probability. Hence, it is sufficient to choose a small $w_{\max }$ - for example, the punctured MB-OFDM code of rate $R_{c}=1 / 2$ [2] has a free distance of 9 , and choosing $w_{\max }=14$ (resulting in a set of $L=242$ error vectors of maximum length $l=60$ ) provides results which are not appreciably different from those obtained using larger $w_{\max }$ values.

We obtained $\mathcal{E}$ by modifying an algorithm for calculating the convolutional code distance spectrum [12] in order to store the code output sequences (i.e., the error vectors $\boldsymbol{e}_{\ell}$ ) in addition to the distance spectrum information. 


\section{B. Pairwise Error Probability}

We consider error events starting in a given position $i$ of the codeword. The set $\zeta$ of allowable starting positions $i$ has size $|\zeta|=R_{c} L_{c}$, and each element $i$ of $\zeta$ is an index $1 \leq$ $i \leq L_{c}$, which is code-dependent. For example, for a code of rate $R_{c}=1 / 2$ the allowable starting positions are $\zeta=$ $\left\{1,3,5, \ldots, L_{c}-1\right\}$.

We consider each error vector $\boldsymbol{e}_{\ell}$ for $1 \leq \ell \leq L$, and form the full error codeword

$$
\boldsymbol{q}_{i, \ell}=[\underbrace{\begin{array}{llll}
0 & 0 & \ldots & 0
\end{array}}_{i-1} \underbrace{\boldsymbol{e}_{\ell}}_{l_{\ell}} \underbrace{\left.\begin{array}{llll}
0 & 0 & \ldots & 0
\end{array}\right]^{T}}_{L_{c}-l_{\ell}-i+1}
$$

of length $L_{c}$ by padding $\boldsymbol{e}_{\ell}$ with zeros on both sides as indicated above. Given the error codeword $\boldsymbol{q}_{i, \ell}$ and given that codeword $c$ is transmitted, the competing codeword is given by

$$
\boldsymbol{v}_{i, \ell}=\boldsymbol{c} \oplus \boldsymbol{q}_{i, \ell} .
$$

Interleaving and modulation results in the vector of QAM symbols

$$
\boldsymbol{z}_{i, \ell}=\mathcal{M}_{\boldsymbol{h}}\left(\boldsymbol{v}_{i, \ell}^{\pi}\right),
$$

where $\boldsymbol{v}_{i, \ell}^{\pi}=\pi\left(\boldsymbol{v}_{i, \ell}\right)$ is the interleaved version of $\boldsymbol{v}_{i, \ell}$.

The PEP for the $\ell$ th error vector starting in the $i$ th position is then given by

$$
\operatorname{PEP}_{i, \ell}=Q\left(\sqrt{\frac{E_{s}}{2 \mathcal{N}_{0}}\left\|\boldsymbol{H}\left(\boldsymbol{x}-\boldsymbol{z}_{i, \ell}\right)\right\|^{2}}\right) .
$$

\section{Per-Realization Performance Analysis}

In this section, we obtain an approximation of the BER for a particular channel realization $\boldsymbol{H}$, which we denote as $P(\boldsymbol{H})$. This method leads naturally to the analysis of the outage performance, which is a useful measure for schemes operating over quasi-static channels.

The PEP for an error vector $\boldsymbol{e}_{\ell} \in \mathcal{E}(1 \leq \ell \leq L)$ with the error event starting in a position $i \in \zeta\left(1 \leq i \leq L_{c}\right)$ is given by (13). The corresponding bit error rate for this event is given by

$$
P_{i, \ell}(\boldsymbol{H})=a_{\ell} \cdot \operatorname{PEP}_{i, \ell}(\boldsymbol{H}) .
$$

Summing over all $L$ error vectors, we obtain an approximation of the BER for starting position $i$ as

$$
P_{i}(\boldsymbol{H})=\sum_{\ell=1}^{L} a_{\ell} \cdot \mathrm{PEP}_{i, \ell}(\boldsymbol{H}) .
$$

We note that (15) can be seen as a standard truncated union bound for convolutional codes (i.e., it is a sum over all error events of Hamming weight less than $\left.\omega_{\max }\right)$. We can tighten this bound by limiting $P_{i}(\boldsymbol{H})$ to a maximum value of $1 / 2$ before averaging over starting positions [13]. Finally, since all allowable starting positions are equally likely, the BER $P(\boldsymbol{H})$ can be written as

$$
P(\boldsymbol{H})=\frac{1}{R_{c} L_{c}} \sum_{i \in \zeta} \min \left[\frac{1}{2}, \sum_{\ell=1}^{L} P_{i, \ell}(\boldsymbol{H})\right] .
$$

Table I contains pseudocode to calculate $P(\boldsymbol{H})$ according to $(16)$.
TABLE I

PSEUDOCODE FOR THE ANALYSIS METHOD.

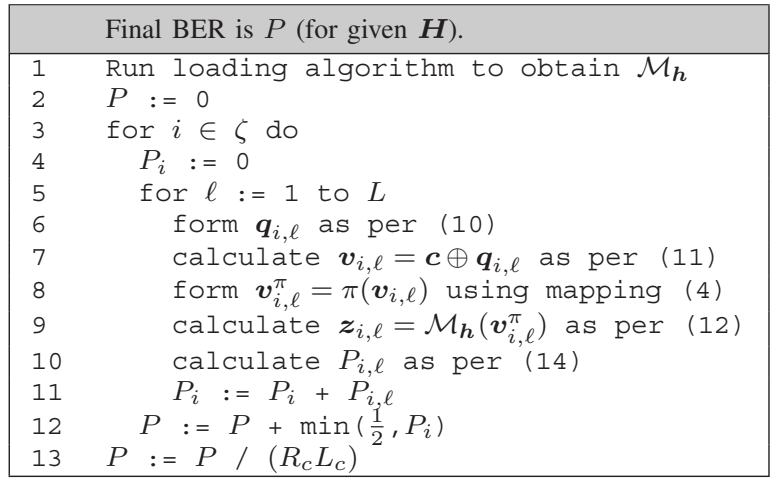

\section{Average and Outage BER}

The average BER can be obtained by averaging (16) over a (large) number $N_{c}$ of channel realizations, where the $i$ th channel realization is denoted by $\boldsymbol{H}_{i}\left(1 \leq i \leq N_{c}\right)$, as

$$
\bar{P}=\frac{1}{N_{c}} \sum_{i=1}^{N_{c}} P\left(\boldsymbol{H}_{i}\right) .
$$

In the quasi-static channel setting it is also often of significant interest to obtain the outage BER performance, i.e., the minimum expected BER performance after excluding some percentage of the worst-performing channel realizations [14, Section III.C-2]. We evaluate (16) for a set of $N_{c}$ channel realizations $\mathcal{H}=\left\{\boldsymbol{H}_{i}, 1 \leq i \leq N_{c}\right\}$. The worst-performing $X \%$ of realizations are considered in outage, and those channel realizations are denoted by $\mathcal{H}_{\text {out }}$. Denoting the remaining $(100-X) \%$ of channel realizations by $\mathcal{H}_{\text {in }}$, the outage BER is given by

$$
P_{\text {out }}=\max _{\boldsymbol{H}_{i} \in \mathcal{H}_{\text {in }}} P\left(\boldsymbol{H}_{i}\right) .
$$

This provides information about the minimum performance that can be expected of the system given the $X \%$ outage rate.

We note that the computational complexity of the analysis scales linearly with $L$ (the size of the set of error vectors) and with $L_{c}$ (the codeword length). Analysis for $L \approx 250$ and $L_{c}=600$ takes only a few seconds per channel realization on a modern PC, and the outage BER for a large number channel realizations (e.g., $N_{c}=500$ ) can be obtained in the time it would take to perform simulations for only one channel realization. Thus, the computational complexity of the analysis is relatively low in comparison to the alternative of performing system simulations. Finally, we note that long packet lengths can be considered without any increase in complexity (if they are segmented into codewords of length $L_{c}$, as is usually the case in practical systems), since the error rate for each codeword will be identical as a result of the quasi-static channel conditions.

\section{Results}

In this section, we present numerical results illustrating the performance analysis presented in Section III. Throughout this 
section, we employ loading schemes with an average $\bar{m}=2$ bits per subcarrier, and allow $m_{i} \in\{0, \ldots, 6\}$ bits per subcarrier. We employ the following loading schemes: the HuguesHartogs algorithm (HHA) [3], [4], the algorithm of Chow, Cioffi and Bingham (CCB) [5], the Piazzo algorithm [6], and the algorithm of Fischer and Huber [7]. Interested readers are referred to the respective papers for the details of each loading algorithm. We also propose a "best solution" loading (BSL) which, for a given channel realization, is obtained by using the analysis to calculate the error rate of each loading algorithm mentioned above, and then selecting the loading with the minimum BER.

We consider two practical OFDM systems: MB-OFDM [2] and $802.11 \mathrm{a} / \mathrm{g}$ [1]. We present the necessary details about each system below.

\section{A. MB-OFDM System and Channel Model}

As the first example OFDM system, we have chosen MBOFDM for high data-rate UWB [2], [15]. MB-OFDM uses 128 subcarriers and operates by hopping over 3 sub-bands (one hop per OFDM symbol) in a predetermined pattern. We will assume that hopping pattern 1 of [2] is used (i.e., the sub-bands are hopped in order). As a result we can consider MB-OFDM as an equivalent 384 subcarrier OFDM system. After disregarding pilot, guard, and other reserved subcarriers, we have $N=300$ data-carrying subcarriers.

Channel coding consists of classical bit-interleaved coded modulation (BICM) [8] with a punctured maximum free distance rate $1 / 3$ constraint length 7 convolutional encoder and a multi-stage block-based interleaver (see [2] for details). In the standard, the interleaved coded bits are mapped to 4QAM symbols using Gray labeling. To maintain the same data rates but decrease the error probability, we instead employ the loading schemes mentioned above with $\bar{m}=2$ bits per subcarrier.

For a meaningful performance analysis of the MB-OFDM proposal, we consider the channel model developed under IEEE 802.15 for UWB systems [16]. The channel impulse response is based on a modified Saleh-Valenzuela model. As well, the entire impulse response undergoes an "outer" lognormal shadowing. The channel impulse response is assumed time invariant during the transmission period of (at least) one packet (see [16] for a detailed description). We consider the UWB channel parameter set referred to as CM1 [16].

\section{B. 802.11a/g System and Channel Model}

The second example system we consider is IEEE $802.11 \mathrm{a} / \mathrm{g}$, which employs 64 subcarriers, of which $N=48$ are used for data transmission [1]. Channel coding is again BICM, with a punctured maximum free distance rate $1 / 2$ constraint length 7 convolutional encoder. We adopt the quasi-static exponentially-decaying multipath Rayleigh fading model used in [10], [17], where the $n$th subcarrier gain of $\boldsymbol{h}$ is given by

$$
h_{n}=\sum_{i=0}^{L_{m}-1} \bar{h}(i) \exp \left(-j \frac{2 \pi n i}{64}\right),
$$



Fig. 1. $10 \%$ Outage BER versus $10 \log _{10}\left(\bar{E}_{b} / \mathcal{N}_{0}\right)$ from analysis (lines) and simulations (markers) for various combinations of code rates and loading algorithms. MB-OFDM system, UWB CM1 channel.

where $L_{m}$ is the number of channel taps and $\bar{h}(i)$ is the $i$ th component of the channel impulse response, modeled as a complex Gaussian random variable [10], [17]

$$
\bar{h}(i) \sim \mathcal{C N}\left(0, \sigma_{0} \exp \left(-i T_{s} / T_{\text {rms }}\right)\right),
$$

where $\sigma_{0}=1-\exp \left(-T_{s} / T_{\mathrm{rms}}\right), T_{s}=50 \mathrm{~ns}$ is the receiver sampling rate, and $T_{\text {rms }}$ is the RMS delay spread of the channel.

\section{Numerical Results}

In Figure 1 we plot the $10 \%$ outage BER $^{2}$ versus $10 \log _{10}\left(\bar{E}_{b} / \mathcal{N}_{0}\right)$ from analysis (lines) as well as the corresponding simulation results (markers) for various combinations of code rates and loading algorithms, for MB-OFDM over the UWB CM1 channel using a set of $N_{c}=100$ channel realizations. We can see that the simulation results confirm the analysis for all considered code rates and loading algorithms, with a maximum difference of $0.4 \mathrm{~dB}$ between simulation and analysis at low BER. We note that obtaining the $10 \%$ outage BER via simulation is very time-consuming due to the need to simulate the system separately for each channel realization. On the other hand, the analysis can be performed quite quickly even for large sets of channels. As a result, we focus exclusively on results from the analysis for the remainder of the paper.

Figure 2 shows the $10 \%$ outage BER versus $10 \log _{10}\left(\bar{E}_{b} / \mathcal{N}_{0}\right)$ for all considered loading algorithms, for the MB-OFDM system with $R_{c}=1 / 2$ over the UWB CM1 channel. We note that for this system and channel model, decent gains of approximately $2 \mathrm{~dB}$ can be obtained via loading. The Piazzo and Fischer-Huber algorithms provide slightly reduced gains. We also note that the performance of HHA and CCB is almost exactly the same, suggesting use

\footnotetext{
${ }^{2}$ The $10 \%$ outage BER is a common performance measure in UWB systems, cf. e.g. [2], [15].
} 


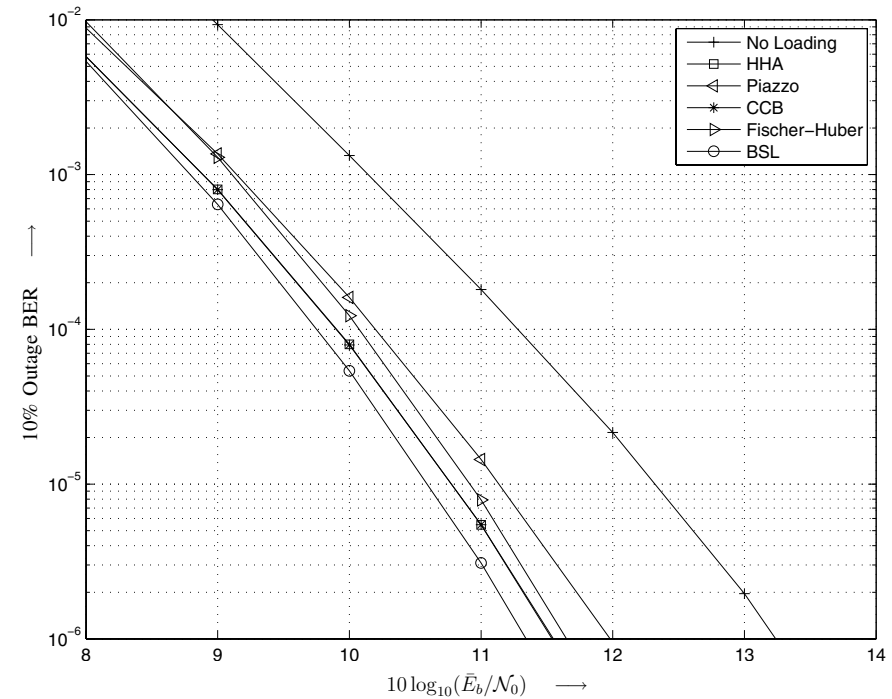

Fig. 2. $10 \%$ Outage BER versus $10 \log _{10}\left(\bar{E}_{b} / \mathcal{N}_{0}\right)$ from analysis for various loading algorithms. MB-OFDM system with $R_{c}=1 / 2$, UWB CM1 channel.

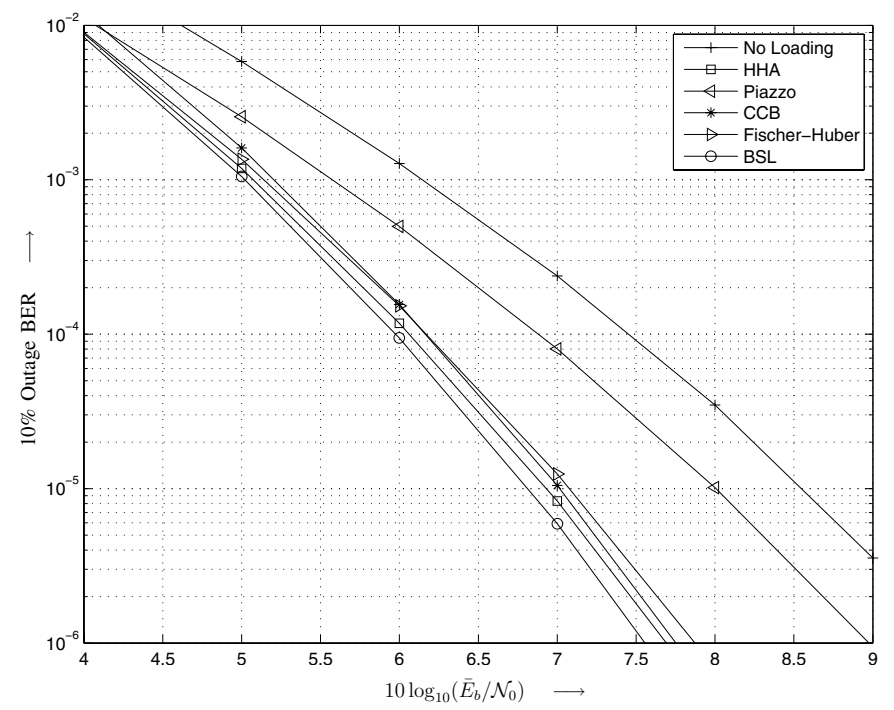

Fig. 3. $10 \%$ Outage BER versus $10 \log _{10}\left(\bar{E}_{b} / \mathcal{N}_{0}\right)$ from analysis for various loading algorithms. $802.11 \mathrm{a} / \mathrm{g}$ WLAN system with $R_{c}=1 / 2$, channel $T_{\mathrm{rms}}=250 \mathrm{~ns}$.

of the latter due to its lower computational complexity [5]. We also note that the BSL results in additional gains over all loading algorithms, at a cost of the increased complexity required to perform the BER analysis for all loadings.

In order to reduce the computational complexity, we also performed the BSL using an error vector set of $L=1$, i.e., we only included the minimum distance error event in the analysis. The results obtained were identical to those in Figure 2, suggesting that reduced-complexity BER estimation with small error vector sets could be an attractive method for loading algorithms based on coded BER, such as BSL.

In Figure 3 we plot the $10 \%$ outage BER versus $10 \log _{10}\left(\bar{E}_{b} / \mathcal{N}_{0}\right)$ for the various loading algorithms, for the $802.11 \mathrm{a} / \mathrm{g}$ WLAN system with $R_{c}=1 / 2$ and channel $T_{\text {rms }}=250$ ns. We see gains of between 1.5 and $2 \mathrm{~dB}$, with



Fig. 4. Cumulative distribution function of loading gain $G=\mathrm{SNR}_{\mathrm{NL}}$ $\mathrm{SNR}_{\text {Loading }}$ required for $\mathrm{BER}=10^{-5}$ for different loading schemes (NL: no loading). MB-OFDM system with $R_{c}=1 / 2$, UWB CM1 channel.

reduced gains for the Piazzo and in particular the FischerHuber algorithms. We again note that the performance of $\mathrm{CCB}$ and HHA algorithms is very similar, and that the BSL can provide additional gains over the other algorithms.

Figure 4 shows the cumulative distribution of the loading gain $G=\mathrm{SNR}_{\mathrm{NL}}-\mathrm{SNR}_{\text {Loading }}$ required for $\mathrm{BER}=10^{-5}$ for different loading schemes (NL: no loading), for the MBOFDM system with $R_{c}=1 / 2$ over the UWB CM1 channel $\left(\mathrm{SNR} \triangleq 10 \log _{10}\left(\bar{E}_{b} / \mathcal{N}_{0}\right)\right)$. Interestingly, we note that there is a small probability that the Piazzo and Fischer-Huber loadings will result in a performance loss (negative gains), while the $\mathrm{CCB}$, HHA, and BSL algorithms always provide a performance gain. We again note that $\mathrm{CCB}$ and HHA algorithms have similar performance. The BSL always results in the highest loading gain. Finally, we note that gains of up to $4 \mathrm{~dB}$ can be expected from loading, while gains of at least $1 \mathrm{~dB}$ can be expected for $50 \%$ of channel realizations.

In Table II we list the relative use of different loading schemes for the BSL (MB-OFDM, $R_{c}=1 / 2$ and $R_{c}=3 / 4$, UWB CM1 channel). Calculating the HHA loading has a high computational complexity compared with the other algorithms, thus, we list the relative use both including and excluding HHA ("w/ HHA" and "w/o HHA", respectively). We note that for $R_{c}=1 / 2, \mathrm{CCB}$ is the most-used algorithm, while for $R=3 / 4$ the Fischer-Huber algorithm is often the best. The Piazzo algorithm is rarely the best loading for either code rate. By comparing the two code rates, we can see that the best loading algorithm is rate dependent, indicating that when deploying coded loaded OFDM systems, some consideration should be given to the loading-coding combination during system design.

Finally, in Table III we list the relative use of different loading schemes for the BSL, for the $802.11 \mathrm{a} / \mathrm{g}$ system with $R_{c}=1 / 2$ and different channel RMS delay spreads $T_{\text {rms }}$. Interestingly, we note that for small $T_{\text {rms }}$ the best loading is 
TABLE II

RELATIVE USE OF DIFFERENT LOADING SCHEMES AS BEST SOLUTION. MB-OFDM, UWB CM1 CHANNEL.

\begin{tabular}{|l|c|c||c|c|}
\cline { 2 - 5 } \multicolumn{1}{c|}{} & \multicolumn{2}{c||}{$\%$ Use $\left(R_{c}=1 / 2\right)$} & \multicolumn{2}{c|}{$\%$ Use $\left(R_{c}=3 / 4\right)$} \\
\hline Loading & w/ HHA & w/o HHA & w/ HHA & w/o HHA \\
\hline \hline No Loading & 0 & 0 & 0 & 0 \\
\hline HHA [3], [4] & 39 & - & 88 & - \\
\hline CCB [5] & 55 & 75 & 8 & 33 \\
\hline Piazzo [6] & 0 & 2 & 0 & 1 \\
\hline Fischer-Huber [7] & 6 & 23 & 4 & 66 \\
\hline
\end{tabular}

TABLE III

RELATIVE USE OF DIFFERENT LOADING SCHEMES AS BEST SOLUTION. 802.11A/G, $R_{c}=1 / 2$, CHANNEL OF (19), (20).

\begin{tabular}{|l|c|c||c|c||c|c|}
\cline { 2 - 7 } \multicolumn{1}{c|}{} & \multicolumn{2}{c||}{$\%$ Use $\left(T_{\text {rms }}=50 \mathrm{~ns}\right)$} & \multicolumn{2}{c||}{$\%$ Use $\left(T_{\text {rms }}=100 \mathrm{~ns}\right)$} & \multicolumn{2}{c|}{ Use $\left(T_{\text {rms }}=250 \mathrm{~ns}\right)$} \\
\hline Loading & w/ HHA & w/o HHA & w/ HHA & w/o HHA & w/ HHA & w/o HHA \\
\hline \hline No Loading & 91 & 94 & 68 & 76 & 17 & 20 \\
\hline HHA [3], [4] & 5 & - & 18 & - & 36 & - \\
\hline CCB [5] & 3 & 4 & 11 & 18 & 31 & 51 \\
\hline Piazzo [6] & 0 & 0 & 0 & 1 & 2 & 3 \\
\hline Fischer-Huber [7] & 1 & 2 & 3 & 5 & 14 & 26 \\
\hline
\end{tabular}

often the same modulation for all subcarriers (no loading). This is a result of the lack of variation in subcarrier channel gains due to the small delay spread. As $T_{\text {rms }}$ increases, the channel gains have more variation and thus there is increased gain from loading. We also note that the best loading is more varied for the WLAN case, indicating again that the choice of a loading algorithm for coded OFDM systems is systemdependent and should be carefully considered during system design.

\section{Conclusions}

In this paper, we have developed a novel analytical method for BER estimation of bit-loaded coded OFDM systems operating over frequency-selective quasi-static channels with nonideal interleaving. To illustrate the application of the proposed analysis, we compared the performance of a number of OFDM bit-loading schemes applied to MB-OFDM for UWB, and to IEEE $802.11 \mathrm{a} / \mathrm{g}$ systems for WLANs.

The results illustrate that the proposed analysis technique provides an accurate estimation of the coded BER of loaded OFDM systems. This allows for system performance analysis without resorting to lengthly simulations. We have also shown that the relative performance of bit-loading algorithms for coded OFDM is system-dependent, and thus some care should be given to the selection of loading algorithms for coded OFDM systems. We have also proposed a "best solution" loading algorithm which selects a loading from amongst a number of candidates and thus guarantees the best performance, at a cost of somewhat higher complexity when performing the loading.

\section{REFERENCES}

[1] IEEE 802.11, "Wireless LAN Medium Access Control (MAC) Physical Layer (PHY) Specifications, Amendment 1: High-Speed Physical Layer in the $5 \mathrm{GHz}$ Band," July 1999.

[2] ECMA, "Standard ECMA-368: High Rate Ultra Wideband PHY and MAC Standard," Dec. 2005, [Online]: http://www.ecmainternational.org/publications/standards/Ecma-368.htm.
[3] J. A.C. Bingham, "Multicarrier Modulation for Data Transmission: An Idea Whose Time Has Come," IEEE Commun. Mag., pp. 5-14, May 1990.

[4] D. Hughes-Hartogs, "Ensemble Modem Structure for Imperfect Transmission Media," U.S. Patents Nos. 4,679,227 (July 1987), 4,731,816 (Mar. 1988), and 4,833,706 (May 1989).

[5] P. S. Chow, J. M. Cioffi, and J. A.C. Bingham, "A Practical Discrete Multitone Transceiver Loading Algorithm for Data Transmission over Spectrally Shaped Channels," IEEE Trans. Commun., vol. 43, no. 2/3/4, pp. 773-775, Feb./Mar./Apr. 1995.

[6] L. Piazzo, "Fast Algorithm for Power and Bit Allocation in OFDM Systems," IEE Electronics Letters, vol. 35, no. 25, pp. 2173-2174, Dec. 1999.

[7] R. F.H. Fischer and J. B. Huber, "A New Loading Algorithm for Discrete Multitone Transmission," in Proc. IEEE GLOBECOM, vol. 1, Nov. 1996, pp. 724-728.

[8] G. Caire, G. Taricco, and E. Biglieri, "Bit-Interleaved Coded Modulation," IEEE Trans. Inform. Theory, vol. 44, no. 3, pp. 927-946, May 1998.

[9] J. G. Proakis, Digital Communications, 4th ed. New York: McGrawHill, 2001.

[10] K.-B. Song, A. Ekbal, S. T. Chung, and J. M. Cioffi, "Adaptive Modulation and Coding (AMC) for Bit-Interleaved Coded OFDM (BICOFDM," IEEE Trans. Wireless Commun., vol. 5, no. 7, pp. 1685-1694, July 2006.

[11] C. Snow, L. Lampe, and R. Schober, "Error Rate Analysis for Coded Multicarrier Systems over Quasi-Static Fading Channels," IEEE Trans. Commun., vol. 55, no. 9, pp. 1736-1746, Sept. 2007.

[12] M. Cedervall and R. Johannesson, "A Fast Algorithm for Computing Distance Spectrum of Convolutional Codes," IEEE Trans. Inform. Theory, vol. 35, no. 6, pp. 1146-1159, Nov. 1989.

[13] E. Malkamäki and H. Leib, "Evaluating the Performance of Convolutional Codes over Block Fading Channels," IEEE Trans. Inform. Theory, vol. 45, no. 5, pp. 1643-1646, July 1999.

[14] E. Biglieri, J. Proakis, and S. Shamai, "Fading Channels: InformationTheoretic and Communications Aspects,' IEEE Trans. Inform. Theory, vol. 44, no. 6, pp. 2619-2692, Oct. 1998.

[15] A. Batra, J. Balakrishnan, G. Aiello, J. Foerster, and A. Dabak, "Design of a Multiband OFDM System for Realistic UWB Channel Environments," IEEE Trans. Microwave Theory Tech., vol. 52, no. 9, pp. 21232138, Sept. 2004.

[16] A. F. Molisch, J. R. Foerster, and M. Pendergrass, "Channel Models for Ultrawideband Personal Area Networks," IEEE Wireless Commun. Mag., pp. 14-21, Dec. 2003.

[17] C. K. Sung and I. Lee, "Adaptive Bit-Interleaved Coded OFDM with Reduced Feedback Information," in Proc. IEEE ICC, 2006, pp. 4630 4635 . 